Publ. RIMS, Kyoto Univ.

40 (2004), 1063-1091

\title{
String and Vortex ${ }^{\dagger}$
}

By

\author{
Toshiya KAWAI*
}

\begin{abstract}
We discuss how the geometry of $D 2-D 0$ branes may be related to Gromov-Witten theory of Calabi-Yau threefolds.
\end{abstract}

\section{$\S 1$. Introduction}

Topological sigma models, first put forward by Witten [34], have long fascinated a number of theoretical physicists and mathematicians. Most remarkably, the task of summing up worldsheet instantons is nowadays elegantly formulated by the theory of Gromov-Witten invariants. Explicit computations of them are still being actively pursued.

It goes without saying that among many possible target spaces Calabi-Yau threefolds have played distinguished roles and are of lasting interest to string theorists. Since the initial appreciation of the significance of D-branes there has been the lingering hope that the Gromov-Witten theory of Calabi-Yau threefolds might be completely rewritten in the language of BPS D-branes.

This contribution is intended for explaining the picture which, to my eye, looks particularly attractive in this regard. This is based on the general philosophy:

The geometry of D2-D0 branes (and not simply D2-branes) provides an alternative description of Gromov-Witten invariants of Calabi-Yau threefolds.

Communicated by T. Kawai. Received December 22, 2003.

2000 Mathematics Subject Classification(s): 81T30, 81T45, 14D21, 14H81, 14 N35

Supported by Grant-in-Aid for Scientific Research No. 13640017 and No. 13135212.

${ }^{\dagger}$ This article is an invited contribution to a special issue of Publications of RIMS commemorating the fortieth anniversary of the founding of the Research Institute for Mathematical Sciences.

*RIMS, Kyoto University, Kyoto 606-8502, Japan. 
What we actually imagine is very simple and intuitive: In analogy with the (generalized) super Kac-Moody algebras we regard the string partition function as the inverse of "denominator function" and interpret the bound state degeneracies of $D 2-D 0$ branes as the "super root multiplicities". The GromovWitten potentials are then extracted from the string partition function. In particular, the variable $y$ measuring $D 0$-charge is related to the genus expansion parameter $\kappa$ of Gromov-Witten theory by $y=\exp (\sqrt{-1} \kappa)$.

This sort of idea was formerly presented in [21] when the Calabi-Yau threefold is elliptically fibered over a Hirzebruch surface. There, an analogy to Borcherds products [2] was pursued. Recall that Borcherds products or their inverses arise in some cases as the denominator functions of generalized (super) Kac-Moody algebras. The first hint of the relevance of Borcherds products to Gromov-Witten theory was given by Harvey and Moore [14]. I will report further progress on the string partition functions of these elliptic Calabi-Yau threefolds elsewhere [20].

So the first purpose of this work is simply to extend the $D 2-D 0$ picture of [21] to general Calabi-Yau threefolds focusing on those aspects which are believed to be independent of any particular details of the threefolds.

Some time ago Gopakumar and Vafa $[10,11]$ proposed an alternative reformulation of Gromov-Witten theory of Calabi-Yau threefolds based on the space-time effective theory interpretation of Gromov-Witten potentials. This claim has been influential but at the same time very mysterious (at least to the author). The second objective of this paper is to discuss how this proposal of Gopakumar and Vafa may actually reconcile with ours.

Quite recently, the relation between singular instantons of $6 d U(1)$ gauge theory and Gromov-Witten theory has been discussed [18, 24] in relation to the topological vertex formalism [1]. This gauge theoretic approach (DonaldsonThomas theory) is probably a dual viewpoint of our $D 2-D 0$ picture in the same way point-like instantons of $4 d U(1)$ gauge theory describe $D 0$-branes. So, as far as the ideology is concerned, these works seem to have some overlaps with [21] and the present work. Nevertheless, there is a marked difference in practice: They discuss the "sum side" with a supply of explicit calculations for local toric Calabi-Yau threefolds using the localization technique whilst we discuss the "product side" inspired from the examples of certain elliptic Calabi-Yau threefolds and the associated Borcherds-like products [21] [20]. It will be very interesting (and necessary!) to investigate the existence of "sum = product" formulas connecting both sides for a general Calabi-Yau threefold. In fact, the topological vertex formalism of [1] seems to allow an intuitive 
understanding in terms of $D 2$-branes and $D 0$-branes. See $\S 6$ for comments on this point.

I am grateful to K. Yoshioka for the collaboration in [21]. I also thank A. Okounkov for kindly pointing out my nonsensical statement in the previous version of the manuscript and for explaining to me the marvelous proposal of him and his collaborators.

\section{Notation.}

For a rational function $f(y)$ of one variable $y$ we define $\iota_{ \pm} f(y) \in \mathbb{C}\left(\left(y^{ \pm 1}\right)\right)$ as follows: $\iota_{+} f(y)$ is the Laurent series of $f(y)$ at $y=0$ and $\iota_{-} f(y)$ is that at $y=\infty$. Consider, for instance, $\phi_{h}(y)=\left(y^{\frac{1}{2}}-y^{-\frac{1}{2}}\right)^{2 h-2}$ for an integer $h \geq 0$. If $h>0$ then $\iota_{+} \phi_{h}(y)$ and $\iota_{-} \phi_{h}(y)$ coincide in $\mathbb{Z}\left[y, y^{-1}\right]$. However,

$$
\iota_{ \pm} \phi_{0}(y)=\sum_{j=1}^{\infty} j y^{ \pm j} \in \mathbb{Z}\left[\left[y^{ \pm 1}\right]\right] .
$$

A Calabi-Yau threefold $X$ is a complex 3 -dimensional smooth projective variety with $c_{1}(X)=0$ and $h^{0,1}(X)=h^{0,2}(X)=0$. We assume that $X$ is polarized by some ample line bundle.

\section{$\S 2$. Why $D 2-D 0$ Rather than $D 2$ ?}

If one ever wishes to connect Gromov-Witten theory of Calabi-Yau threefolds to some sort of BPS $D$-brane counting, one might think that $D 2$-branes alone are relevant since Gromov-Witten theory is concerned with curve counting problems. However, this is too naive and even misleading. What needs to be emphasized is that in any attempt of this sort we have to incorporate the effects of $D 0$-branes in addition to $D 2$-branes.

Take as an example the case of a resolved conifold, the total space of $\mathcal{O}_{\mathbb{P}^{1}}(-1) \oplus \mathcal{O}_{\mathbb{P}^{1}}(-1)$. Since the $\mathbb{P}^{1}$ in the resolved conifold is rigid and the Jacobian of $\mathbb{P}^{1}$ is just a point, the moduli space of $D 2$-brane wrapping around the $\mathbb{P}^{1}$ is also a point. If $D 2$-branes were the only relevant $D$-branes, this would mean that the Gromov-Witten theory of the resolved conifold was trivial. This is simply absurd given the result of [4].

The origin for the necessity of $D 0$-branes may be roughly as follows. Let us suppose that we are trying to answer the problem of counting curves in a Calabi-Yau threefold $X$. There could be several different approaches according what we mean by counting.

In a crude approach by $D$-branes one may make curves "charged" by putting line bundles, (or more generally, rank one torsion-free sheaves) on them 
and then claim, to within signs, the Euler-Poincaré characteristics of the moduli spaces of such sheaves (regarded as torsion sheaves on $X$ ) with fixed $D$-brane charges as the "numbers of curves". Apparently, in this naive approach we are concerned with $D 2$-branes alone.

In Gromov-Witten theory, on the other hand, one tries to "count holomorphic maps" from worldsheet connected curves to target curves in $X$ and regard this as a good substitute of directly counting curves in $X$. However, this very substitution introduces some well-known complications. One of them is the so-called multi-covering effect. This is rather innocuous since we know more or less how to handle it. Another complication, which seems to be more difficult, is related to conformal invariance of the holomorphic map equation and is known as bubbling phenomena. In order to have a nice intersection theory one must compactify the moduli space of holomorphic maps. For this we have to include degenerate contributions of bubble trees (bubbling of bubbling of ...). It should be precisely for this reason that we have to modify the naive $D 2$-brane approach by including $D 0$-branes when one attempts to rewrite Gromov-Witten theory in terms of $D$-branes. An intuitive picture of $D 0$-branes bound to a D2-brane is that of vortices. So morally speaking, bubble trees turn into vortices.

\section{§3. String Partition Function}

Suppose that we are given a super Kac-Moody algebra. Let $Q_{+}$denote the additive semigroup generated by simple roots. We write $\alpha>0$ iff $\alpha \in Q_{+} \backslash\{0\}$. Any $\alpha>0$ is either even or odd. Consider

$$
\begin{aligned}
\Gamma: & =-\sum_{\alpha>0, \alpha: \text { even }} \operatorname{mult}(\alpha) e^{-\alpha}+\sum_{\alpha>0, \alpha: \text { odd }} \operatorname{mult}(\alpha) e^{-\alpha} \\
& =-\sum_{\alpha>0} \operatorname{smult}(\alpha) e^{-\alpha}
\end{aligned}
$$

so that $\alpha>0$ is a positive root iff $\operatorname{smult}(\alpha) \neq 0$. We have

$$
\exp \left(-\sum_{k=1}^{\infty} \frac{1}{k} \psi^{k}(\Gamma)\right)=\prod_{\alpha>0}\left(1-e^{-\alpha}\right)^{-\operatorname{smult}(\alpha)},
$$

where $\psi^{k}$ is the $k$-th Adams operation sending $e^{-\alpha}$ to $e^{-k \alpha}$. The right hand side is the inverse of the denominator function in the product form.

In a nutshell, what we intend to do is to make the analogy of this relation in studying a Calabi-Yau threefold $X$. We express any element of $H_{2}(X, \mathbb{Z}) \oplus$ 
$H_{0}(X, \mathbb{Z})$ as $(\beta, j)$ where $\beta \in H_{2}(X, \mathbb{Z})$ and $j \in \mathbb{Z} \cong H_{0}(X, \mathbb{Z})$. Denote by $N_{+}$ the additive semigroup of classes of effective 1-cycles ("holomorpic curves") on $X$. In other words, $N_{+}$is the intersection of the Mori cone with $H_{2}(X, \mathbb{Z})$. We write $\beta>0$ iff $\beta \in N_{+} \backslash\{0\}$. Similarly, for $(\beta, j) \in H_{2}(X, \mathbb{Z}) \oplus H_{0}(X, \mathbb{Z})$ we write $(\beta, j)>0$ iff

$$
[\beta>0] \text { or } \quad[\beta=0 \text { and } j>0] .
$$

Assume that there is a suitable $\mathbb{Z}_{2}$-grading so that any $(\beta, j)>0$ is either even or odd. Now let us suppose in analogy with $\Gamma$ the existence of a formal sum:

$$
\Gamma_{X}=-\sum_{(\beta, j)>0} \operatorname{smult}(\beta, j) \mathbf{q}^{\beta} y^{j},
$$

where $\mathbf{q}^{\beta}$ is a formal symbol satisfying $\mathbf{q}^{\beta} \mathbf{q}^{\beta^{\prime}}=\mathbf{q}^{\beta+\beta^{\prime}}$. In the proposal below, $\operatorname{smult}(\beta, j)$ is in $\mathbb{Z} / 2$ rather than in $\mathbb{Z}$. This is to match up with the normalization of Gromov-Witten potentials.

We next introduce the formal truncated ${ }^{1}$ free energy

$$
F:=-\sum_{k=1}^{\infty} \frac{1}{k} \psi^{k}\left(\Gamma_{X}\right),
$$

where $\psi^{k}$ is again the $k$-th Adams operation sending $\mathbf{q}^{\beta} y^{j}$ to $\mathbf{q}^{k \beta} y^{k j}$.

As the inverse of the "denominator function" 2 we are led to consider the following formal product

$$
\left.Z=\prod_{(\beta, j)>0}\left(1-\mathbf{q}^{\beta} y^{j}\right)^{-\operatorname{smult}(\beta, j}\right) .
$$

We might call $Z$ as the formal truncated string partition function. However the expected relation " $Z=\exp (F)$ " is rather problematic since, in the formal sum (3.4), $j$ runs over all integers when $\beta>0$ so that powers of $F$ are not welldefined in general. Thus $F$ and $Z$ in the above should be interpreted at best as motivating expressions. In the following we will introduce regularized versions $\tilde{Z}$ and $\tilde{F}$ related by $\tilde{Z}=\exp (\tilde{F})$. (These are better behaved but somewhat lose direct analogy with super Kac-Moody algebras.)

Remark. Those who are familiar with Borcherds products and their relations to surfaces, say, $K 3$ surfaces will recognize that we are trying to cook

\footnotetext{
${ }^{1}$ Here truncated means that the "Weyl vector" is truncated. See Remark 5.

${ }^{2}$ The reason we consider the inverse is that we adopt the usual rule: "even = bosonic" \& "odd = fermionic".
} 
up a similar story for Calabi-Yau threefolds here. A novel and distinct point is the introduction of $H_{0}(X, \mathbb{Z})$ in addition to $H_{2}(X, \mathbb{Z})$. I intend to further discuss this similarity with Borcherds products in [20].

For the above analogy to be anything useful we have to know $\Gamma_{X}$ from the geometry of $X$. Our basic expectation is that $\operatorname{smult}(\beta, j)$ should be, in some way or another, identified with the (super) degeneracy of bounded $D 2$ $D 0$ branes in $X$ with a fixed $D 2-D 0$ charge $(\beta, j)$. Therefore, what needs to be done is the geometrical understanding of $D 2-D 0$ bound systems.

\section{$\S 4 . \quad D 2-D 0$ and $D 2$ Moduli Spaces}

We first recall the gentlemen's agreement [15] that even-dimensional $D$ branes are related to coherent sheaves and their $D$-brane charges are determined by Mukai vectors $[25,26]$. We define the $D$-brane charge $Q(\varepsilon)$ of a coherent sheaf $\mathcal{E}$ on $X$ by $Q(\mathcal{E})=v(\mathcal{E}) \cap[X]$ where $v(\mathcal{E})=\operatorname{ch}(\mathcal{E}) \sqrt{\operatorname{td}(X)}$ is the Mukai vector of $\mathcal{E}$. We express $Q(\mathcal{E})$ in the form $\left(Q_{6}, Q_{4}, Q_{2}, Q_{0}\right)$ where $Q_{2 i} \in H_{2 i}(X, \mathbb{Q})$. Note that if the $D$-brane charge is of the form $\left(0,0, Q_{2}, Q_{0}\right)$ then $\left(Q_{2}, Q_{0}\right)$ is actually in $H_{2}(X, \mathbb{Z}) \oplus H_{0}(X, \mathbb{Z})$. (There is no Witten effect.) For instance, suppose that $i: C \hookrightarrow X$ is a smooth irreducible curve of genus $g$ and $L \rightarrow C$ is a line bundle of degree $d$, then $Q\left(i_{*} L\right)=(0,0,[C], \chi(C, L))=(0,0,[C], d+1-g)$. This is the $D$-brane charge of a $D 2$-brane singly wrapping around $C$ bound with $d D 0$-branes. In this paper any $D 2$-brane is always singly wrapping by allowing non-reduced curves.

If the $D$-brane charge (or equivalently the Mukai vector) is fixed, the Hilbert polynomial is also fixed since $X$ is assumed to be polarized. So it makes sense to consider the moduli space $\mathcal{M}(Q)$ of semi-stable coherent sheaves on $X$ with a fixed $D$-brane charge $Q$. For simplicity let us suppose that $\mathcal{M}(Q)$ consists only of stable sheaves. One well-known fact about $\mathcal{M}(Q)$ is that its expected dimension $\sum_{i=0}^{3}(-1)^{i+1} \operatorname{dim}_{\operatorname{Ext}_{0}^{i}}(\mathcal{E}, \mathcal{E})$ vanishes because of the Serre duality where $\mathcal{E} \in \mathcal{M}(Q)$ and $\operatorname{Ext}_{0}^{i}$ is the trace free part of Ext ${ }^{i}$. By stability, $\operatorname{Ext}_{0}^{0}(\mathcal{E}, \mathcal{E}) \cong\{0\} \cong \operatorname{Ext}_{0}^{3}(\mathcal{E}, \mathcal{E})$ and by $h^{0,1}(X)=h^{0,2}(X)=0$, we have $\operatorname{Ext}^{i}(\mathcal{E}, \mathcal{E})=\operatorname{Ext}_{0}^{i}(\mathcal{E}, \mathcal{E})(i=1,2)$. The zero-dimensional virtual moduli cycle was constructed in [31]. Its degree or virtual length $\lambda(\mathcal{M}(Q)) \in \mathbb{Z}$ serves as the "number of sheaves". The Zariski tangent space of $\mathcal{M}(Q)$ at $\mathcal{E}$ is given by $\operatorname{Ext}^{1}(\mathcal{E}, \mathcal{E})$ and the obstruction space is $\operatorname{Ext}^{2}(\mathcal{E}, \mathcal{E})$. Hence the Serre duality tells us that if $\mathcal{M}(Q)$ is smooth, the obstruction sheaf is the cotangent bundle of $\mathcal{M}(Q)$ and $\lambda(\mathcal{M}(Q))=(-1)^{\operatorname{dim} \mathcal{M}(Q)} \chi(\mathcal{M}(Q))$.

One can also consider the Hilbert scheme $\operatorname{Hilb}(Q)$ with a fixed $D$-brane charge $Q$. As usual, we have $\operatorname{Hilb}(Q)=\mathcal{M}\left(Q\left(\mathcal{O}_{X}\right)-Q\right)$ by considering ideal 
sheaves. The moduli space of possibly disconnected $D 2$-branes and $D 0$-branes, which we call the total $D 2-D 0$ moduli space, is given by

$$
\coprod_{\left(Q_{2}, Q_{0}\right)} \operatorname{Hilb}\left(0,0, Q_{2}, Q_{0}\right),
$$

where $\left(Q_{2}, Q_{0}\right)$ runs over all possible values. At first sight (4.1) seems not to deserve its name. For instance, if an isolated $D 2$-brane is singly wrapping around a smooth curve $C \subset X$, there should exist degrees of freedom in how it wraps, namely the Jacobian $J(C)$. On the other hand in the Hilbert scheme $C$ is allowed to carry only $\mathcal{O}_{C}$. A possible resolution of this puzzle may be as follows. First of all if a $D 2$-brane wrapping singly around $C$ is bounded to several $D 0$-branes, the moduli space of this bound system is given by a symmetric product of $C$ (as we recall later). So this part of the moduli space is directly related to the Hilbert scheme (4.1). The pure $D 2$-brane moduli space $J(C)$ is actually related to the symmetric products of $C$ through the AbelJacobi maps. So, once we start to consider both $D 2$-branes and $D 0$-branes simultaneously, we should forget about $J(C)$ and only consider the symmetric products of $C$ as fundamental in order not to overcount the degrees of freedom. A more explanation is given below about how pure $D 2$-brane moduli spaces are related to the moduli spaces of bounded $D 2-D 0$ branes. (This is not unrelated to the upcoming interpretation of the Gopakumar-Vafa proposal.)

A relation between $\lambda(\mathcal{M}(Q))$ and Gromov-Witten invariants was already hinted in [31]. A connection to the Gopakumar-Vafa invariant was conjectured in [13]. Recently, a striking connection of $\lambda(\operatorname{Hilb}(Q))$ to Gromov-Witten theory has been proposed in [24].

We expect that $\Gamma_{X}$ is related to (4.1) but exactly describing this relation seems to be a very difficult problem at the moment. Only a limited attempt is given below. Let us call $\beta \in N_{+}$of simple class if its arithmetic genus $g(\beta)$ is non-negative and for each integer $d \geq 0$ there exist a suitable $D 2-D 0$ bound moduli space $\mathfrak{M}_{\beta, d}$ and a $D 2$ moduli space $\mathfrak{N}_{\beta, d}$ whose properties we spell out in the following. We assume that $\beta=0$ is of simple class and set $g(0)=0$.

The $D 2-D 0$ bound moduli space $\mathfrak{M}_{\beta, d}$ describes a $D 2$-brane wrapping around a curve of class $\beta$ bound to $d D 0$-branes. In particular we expect an intimate connection between $\mathfrak{M}_{\beta, d}$ and $\operatorname{Hilb}(0,0, \beta, d+1-g(\beta))$. We assume that $\mathfrak{M}_{\beta, d}$ is smooth and connected. As we will see shortly, it seems natural to have

$$
\mathfrak{M}_{0, d}=X \times \mathbb{P}^{d}
$$

We set $S_{\beta}:=\mathfrak{M}_{\beta, 0}$ for convenience. This is the moduli space of the supports 
of $D 2$-branes. Note that $S_{0}=X$ by (4.2). There should exist a morphism $\mathfrak{M}_{\beta, d} \rightarrow S_{\beta}$ and

$$
\operatorname{dim} \mathfrak{M}_{\beta, d}=S_{\beta}+d
$$

Moreover, there will be the universal family $\mathcal{C}_{\beta} \rightarrow S_{\beta}$ which is a flat family of curves of class $\beta$ and of arithmetic genus $g(\beta)$. In particular we expect

$$
\mathcal{C}_{0}=X \times \mathbb{P}^{1}
$$

where the morphism $\mathcal{C}_{0} \rightarrow S_{0}$ is the projection. Intuitively speaking, $\mathcal{C}_{\beta} \rightarrow S_{\beta}$ is the family of curves around which $D 2$-branes are singly wrapping. In favorable situations it is tempting to identify $\mathfrak{M}_{\beta, d}$ with the relative Hilbert scheme of points on curves

$$
\mathcal{C}_{\beta}^{[d]}:=\operatorname{Hilb}_{\mathcal{C}_{\beta} / S_{\beta}}^{d}
$$

Since we assumed that $\mathfrak{M}_{\beta, d}$ is smooth, our concern is limited to a $\operatorname{smooth} \mathcal{C}_{\beta}^{[d]}$.

Let us explain why (4.2) and (4.4) seem natural. We regard $\mathfrak{M}_{0, d}$ as the moduli space of the bound system of a $D 2$-brane and $d D 0$-branes in the limit where the support curve of the $D 2$-brane is shrinking to a point. What does the curve look like? Since we do not expect any D2-brane degree of freedom in the end, the Jacobian of the curve must be a point. So the curve will be a $\mathbb{P}^{1}$. With the vortex interpretation given in [21] and to be recalled later, the moduli space of the $D 2$-brane wrapping around $\mathbb{P}^{1}$ and $d D 0$-branes sticked to it should be given by the $d$-th symmetric product of $\mathbb{P}^{1}$ or $\mathbb{P}^{d}$. Therefore if we take into account the location of the shrinking $\mathbb{P}^{1}$ in $X$, we are led to (4.2) and (4.4).

Let us turn to the properties of the $D 2$ moduli space. We assume that $\mathfrak{N}_{\beta, d}$ is smooth and connected. There should exist a natural morphism $\mathfrak{N}_{\beta, d} \rightarrow S_{\beta}$. Intuitively, $\mathfrak{N}_{\beta, d}$ is the moduli space of $D 2$-branes singly wrapping around fibers of $\mathcal{C}_{\beta} \rightarrow S_{\beta}$. We expect an intimate connection between $\mathfrak{N}_{\beta, d}$ and $\mathcal{M}(0,0, \beta, d+1-g(\beta))$. We furthermore expect that $\mathfrak{N}_{\beta, d}$ is independent of $d$ and is isomorphic to $\mathfrak{N}_{\beta}:=\mathfrak{N}_{\beta, 0}$. We should have $\mathfrak{N}_{0}=X$. Hence $\mathfrak{N}_{0} \rightarrow S_{0}$ is the identity map. We require the existence of a commutative diagram

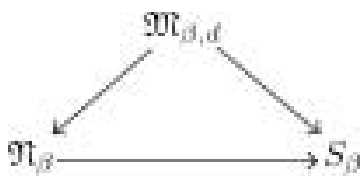


For $\beta=0$ we have (4.6) by obvious morphisms. For $d=0, \mathfrak{M}_{\beta, 0}=S_{\beta} \rightarrow$ $\mathfrak{N}_{\beta}$ may be viewed as taking a section.

If $\mathfrak{M}_{\beta, d}=\mathcal{C}_{\beta}^{[d]}$, one may take

$$
\mathfrak{N}_{\beta, d}=\overline{\operatorname{Pic}}_{\mathcal{C}_{\beta} / S_{\beta}}^{d}, \quad \mathfrak{N}_{\beta}=\overline{\operatorname{Pic}}_{\mathcal{C}_{\beta} / S_{\beta}}^{0}=: \overline{\mathcal{J}}_{\beta},
$$

where $\overline{\mathrm{Pic}}_{\mathcal{C}_{\beta} / S_{\beta}}^{d}$ is the relative compactified Picard scheme and $\overline{\mathcal{J}}_{\beta}$ is the relative compactified Jacobian. Then the diagram (4.6) is replaced by

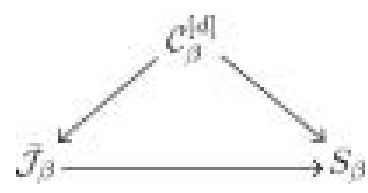

The morphism $\mathcal{C}_{\beta}^{[d]} \rightarrow \overline{\mathcal{J}}_{\beta}$ is just the Abel-Jacobi map.

We should have

$$
\operatorname{dim} \mathfrak{N}_{\beta}=\operatorname{dim} S_{\beta}+g(\beta) .
$$

This ends the list of what we demand for $\beta$ to be of simple class.

We now turn to our proposal on the structure of $\Gamma_{X}$ :

Proposal 4.1. By taking into account charge conjugation symmetry, we have

$$
\begin{aligned}
2 \Gamma_{X} & =\sum_{d=0}^{\infty} \nu(0, d) y^{d+1} \\
& +\sum_{\beta>0} \sum_{d=0}^{\infty} \nu(\beta, d)\left(y^{d+1-g(\beta)}+y^{-(d+1-g(\beta))}\right) \mathbf{q}^{\beta}
\end{aligned}
$$

where $\nu(\beta, d) \in \mathbb{Z}$ and if $\beta$ is of simple class $\nu(\beta, d)=\epsilon(\beta) \chi\left(\mathfrak{M}_{\beta, d}\right)$ with

$$
\epsilon(\beta)=(-1)^{\operatorname{dim} S_{\beta}+g(\beta)}=(-1)^{\operatorname{dim} \mathfrak{N}_{\beta} .}
$$

This looks like the most natural extension of what we conceived in [21]. The reason why we choose the particular sign factor (4.11) is explained below by comparison with Gromov-Witten theory. Note that even if $\mathfrak{M}_{\beta, d}$ is nonempty, $\chi\left(\mathfrak{M}_{\beta, d}\right)$ can vanish. In our analogy with super Kac-Moody algebras this is related to the vanishing of $\operatorname{smult}(\beta, j)$ when $(\beta, j)$ is not a "positive root".

At the moment we do not know how exactly $\nu(\beta, d)$ should be described geometrically when $\beta$ is other than of simple class. 


\section{§5. Gromov-Witten Potentials}

To make contact with Gromov-Witten theory we further postulate

Assumption 5.1. For each $\beta \in N_{+}$there exists a rational function $h_{\beta}(y)$ with inversion symmetry $h_{\beta}(y)=h_{\beta}\left(y^{-1}\right)$ such that

$$
\sum_{d=0}^{\infty} \nu(\beta, d) y^{ \pm(d+1-g(\beta))}=\iota_{ \pm} h_{\beta}(y) .
$$

If $\beta$ is of simple class, we introduce $f_{\beta}(y)$ by $h_{\beta}(y)=\epsilon(\beta) f_{\beta}(y)$, namely,

$$
\sum_{d=0}^{\infty} \chi\left(\mathfrak{M}_{\beta, d}\right) y^{ \pm(d+1-g(\beta))}=\iota_{ \pm} f_{\beta}(y) .
$$

Moreover we have the expansion of the form

$$
h_{\beta}(y)=-\sum_{g=0}^{\infty} r_{\beta}^{g} \kappa^{2 g-2},
$$

where $y=\exp (\sqrt{-1} \kappa)$ and $r_{\beta}^{g} \in \mathbb{Q}$.

One then observes that

$$
2 \Gamma_{X}=\iota_{+} h_{0}(y)+\sum_{ \pm} \iota_{ \pm} \sum_{\beta>0} h_{\beta}(y) \mathbf{q}^{\beta} .
$$

So far $\mathbf{q}^{\beta}$ has been a formal symbol, but in Gromov-Witten theory we should like to set $\mathbf{q}^{\beta}=\exp (\omega \cap \beta)$ where $\omega$ is the complexified Kähler form of $X$. Therefore, motivated by $(5.4)$, we introduce

$$
\widetilde{\Gamma}_{X}=\frac{1}{2} h_{0}(y)+\sum_{\beta>0} h_{\beta}(y) \mathbf{q}^{\beta},
$$

and interpret this as a power series expansion in $q_{i}:=\exp \left(\omega \cap \beta_{i}\right)(i=$ $\left.1, \ldots, h^{1,1}(X)\right)$. Here $\beta_{1}, \ldots, \beta_{h^{1,1}(X)} \in H_{2}(X, \mathbb{Z})$ are generators of the Mori cone. In addition one may introduce the truncated free energy by

$$
\tilde{F}:=-\sum_{k=1}^{\infty} \frac{1}{k} \psi^{k}\left(\widetilde{\Gamma}_{X}\right),
$$

and the truncated string partition function $\tilde{Z}:=\exp (\tilde{F})$. Then, if $\left|\mathbf{q}^{\beta} y^{d+1-g(\beta)}\right|$ $\ll 1$ for all $\beta \geq 0$ and $d \geq 0$, we have an infinite product representation:

$$
\tilde{Z}=\prod_{\beta \geq 0} \prod_{d=0}^{\infty}\left(1-\mathbf{q}^{\beta} y^{d+1-g(\beta)}\right)^{\mu(\beta, d)},
$$


where

$$
\mu(\beta, d)= \begin{cases}\frac{1}{2} \nu(0, d), & \beta=0 \\ \nu(\beta, d), & \beta>0\end{cases}
$$

By (4.2) we have $\chi\left(\mathfrak{M}_{0, d}\right)=(d+1) \chi(X)$. Hence we have $\mu(\beta, d) \in \mathbb{Z}$ because of $\chi(X) \in 2 \mathbb{Z}$.

To discuss the relation to Gromov-Witten theory we want to set $y=$ $\exp (\sqrt{-1} \kappa)$ with $\kappa \in \mathbb{R}$ and $|\kappa|$ very small and interpret $\kappa$ as the genus expansion parameter. As above we always assume $\left|\mathbf{q}^{\beta}\right| \ll 1$ for $\beta>0$ in the following ${ }^{3}$. For such expansions in $\kappa$ to be possible, the truncated free energy needs a bit of regularization:

$$
\tilde{F}^{s}:=-\sum_{k=1}^{\infty} \frac{1}{k^{s}} \psi^{k}\left(\widetilde{\Gamma}_{X}\right) \quad(s \in \mathbb{C}, \Re(s)>1) .
$$

Let us introduce $F_{g}^{s}$ via the expansion $\tilde{F}^{s}=\sum_{g=0}^{\infty} \kappa^{2 g-2} F_{g}^{s}$ and define

$$
F_{g}:=\lim _{s \rightarrow 1} F_{g}^{s}, \quad(g \neq 1), \quad F_{1}:=\lim _{s \rightarrow 1}\left[F_{1}^{s}-\frac{1}{2} r_{0}^{1}\left(\frac{1}{s-1}+\gamma_{\mathrm{em}}\right)\right],
$$

where $\gamma_{\mathrm{em}}$ is the Euler-Mascheroni constant.

Conjecture 5.1. The Gromov-Witten potentials $\mathbf{F}_{g}(g \geq 0)$ of $X$ are given by

$$
\mathbf{F}_{0}=F_{0}^{\mathrm{cl}}+F_{0}, \quad \mathbf{F}_{1}=F_{1}^{\mathrm{cl}}+F_{1}, \quad \mathbf{F}_{g}=F_{g} \quad(g>1),
$$

where

$$
F_{0}^{\mathrm{cl}}=\frac{1}{3 !} \int_{X} \omega^{3}, \quad F_{1}^{\mathrm{cl}}=-\frac{1}{24} \int_{X} c_{2}(X) \omega .
$$

Remark. In seeking analogy with super Kac-Moody algebras $\kappa^{-2} F_{0}^{\mathrm{cl}}+$ $F_{1}^{c l}$ should be interpreted as (the negative of) the "Weyl vector". This part is quite delicate and important.

In fact by using (5.3) we find that

$$
F_{g}=\frac{1}{2} r_{0}^{g} \zeta^{*}(3-2 g)+\sum_{\beta>0} r_{\beta}^{g} \operatorname{Li}_{3-2 g}\left(\mathbf{q}^{\beta}\right),
$$

where $\zeta^{*}(s):=\zeta(s)$ if $s \neq 1$ and $\zeta^{*}(1):=0$. This is an expected form.

\footnotetext{
${ }^{3}$ However, as is often the case in Gromov-Witten theory we neglect the issue of convergence.
} 
Let us give some evidence for our choice (4.11). Note first that $\epsilon(0)=$ $(-1)^{3}=-1$ and

$$
f_{0}(y)=\frac{\chi(X)}{\left(y^{\frac{1}{2}}-y^{-\frac{1}{2}}\right)^{2}} .
$$

This enables us to calculate $r_{0}^{g}$ explicitly. For $g=0$, we have $\frac{1}{2} r_{0}^{0} \zeta(3)=$ $-\frac{1}{2} \chi(X) \zeta(3)$. This is familiar to us. As for $g>1$, the constant map contribution is correctly reproduced:

$$
\frac{1}{2} r_{0}^{g} \zeta(3-2 g)=\frac{1}{2} \chi(X)(-1)^{g} \int_{\overline{\mathcal{M}}_{g, 0}}\left(\lambda_{g-1}\right)^{3}, \quad(g>1),
$$

where $\overline{\mathcal{M}}_{g, 0}$ is the Deligne-Mumford moduli stack of stable curves of arithmetic genus $g$ without marked points and $\lambda_{g-1}$ is the $(g-1)$-th Chern class of the Hodge bundle over $\overline{\mathcal{M}}_{g, 0}$. The relation (5.15) was conjectured physically in [10] from $M$-theory interpretation and in [22] from duality to heterotic string. A mathematical proof of (5.15) was given in [4]. An argument here from a purely $D 2-D 0$ point of view is new.

Suppose that $\beta$ is of simple class and that both $\mathcal{C}_{\beta}$ and $S_{\beta}$ are smooth. Assume that all the fibers of $\mathcal{C}_{\beta} \rightarrow S_{\beta}$ are smooth irreducible curves of genus $g(\beta)$. Then the vortex interpretation explained below shows that

$$
f_{\beta}(y)=\chi\left(S_{\beta}\right)\left(y^{\frac{1}{2}}-y^{-\frac{1}{2}}\right)^{2 g(\beta)-2} .
$$

If $\epsilon(\beta)=(-1)^{\operatorname{dim} S_{\beta}+g(\beta)}$ we see that $r_{\beta}^{g(\beta)}=(-1)^{\operatorname{dim} S_{\beta}} \chi\left(S_{\beta}\right)=\int_{S_{\beta}} c_{\text {top }}\left(T_{S_{\beta}}^{\vee}\right)$. This is consistent with Gromov-Witten theory.

\section{§6. Comments on the "Sum Side"}

Not infrequently we encounter non-trivial formulas of the form

$$
\text { sum=product. }
$$

So far we have been discussing the product side. It is natural to ask what the sum side looks like. Obviously, the sum should be taken over potentially all the $D 2-D 0$ states. So the total $D 2-D 0$ moduli space (4.1) will be relevant. Quite recently $[18,24]$, in an attempt to understand the formalism of the topological vertex for local Calabi-Yau threefolds, the connection between singular instantons of $6 d U(1)$ gauge theory and Gromov-Witten theory has been studied. This is expected to be another description of our $D 2-D 0$ picture studied on the sum side. In particular, [24] treats local Calabi-Yau threefolds and evaluates, 
by the localization technique, the generating function of $\lambda(\mathcal{M}(Q))$ for the moduli spaces of ideal sheaves $\mathcal{M}(Q)=\operatorname{Hilb}\left(Q\left(\mathcal{O}_{X}\right)-Q\right)$. This is a very explicit and remarkable calculation.

In fact the topological vertex formalism $[1,29]$ fits quite nicely into the picture of $D 2-D 0$ branes. For a local toric Calabi-Yau threefold, the relevant sum is reduced by localization to the one over the torus-fixed configurations of $D 2-D 0$ branes. In the approach of [1] one writes the diagram consisting of edges and trivalent vertices. Each internal edge corresponds to a $\left(\mathbb{C}^{\times}\right)^{2}$ fixed $\mathbb{P}^{1}$. Each vertex corresponds to a $\left(\mathbb{C}^{\times}\right)^{3}$-fixed point and is the north or south poles of the $\mathbb{P}^{1}$ 's of the internal edges emanating from it. It is clear that the torus-fixed configurations of $D 2-D 0$ branes are such that $D 2$-branes are localized to the $\left(\mathbb{C}^{\times}\right)^{2}$-fixed $\mathbb{P}^{1}$ 's (the internal edges) and $D 0$-branes are localized to the $\left(\mathbb{C}^{\times}\right)^{3}$-fixed points (the vertices). The way several $D 2$-branes are localized to the internal edge $\mathbb{P}^{1}$ 's must be treated scheme-theoretically and described by assigning a $2 d$ Young diagram to each edge. Similarly, the way $D 0$-branes accumulate to the vertex points must also be treated schemetheoretically and described by assigning a $3 d$ Young diagram to each vertex. However the configurations of $D 0$-branes and those of $D 2$-branes are not independent. How these are actually linked is specified by the rule of the topological vertex.

It should be mentioned that having a nice sum expression does not a priori guarantee the connection to Gromov-Witten theory. For such to exist "sum = product" will probably have to hold. For super (generalized) KacMoody algebras, this problem is related to finding the set of positive roots among $Q_{+}$, which in general is a subtle and difficult task.

Obviously, if our "product" proposal and the "sum" proposal (GW/DT correspondence) of [24] are consistent, $\{\nu(\beta, d)\}$ and $\left\{\lambda\left(\operatorname{Hilb}\left(0,0, Q_{2}, Q_{0}\right)\right\}\right.$ must be related. This looks plausible since both are concerned with counting $D 2$ and $D 0$ branes in $X$. However, the precise geometrical characterization of $\{\nu(\beta, d)\}$ is yet to be found.

\section{$\S 7 . \quad$ Comparison with Gopakumar-Vafa Proposal}

In [11], Gopakumar and Vafa asserted that the D2-brane moduli spaces of a Calabi-Yau threefold $X$ have some characteristic properties. We considered the $D 2$ moduli space $\mathfrak{N}_{\beta}$ when $\beta$ is of simple class and assumed that $\mathfrak{N}_{\beta}$ is smooth. To recall the Gopakumar-Vafa proposal, let us temporarily lift the smoothness condition of $\mathfrak{N}_{\beta}$. In this slightly more general context, we cast their claim taking into account the suggestion of [13] as follows: 
Let I $H^{*}\left(\mathfrak{N}_{\beta}\right)$ denote the intersection cohomologies of $\mathfrak{N}_{\beta}$. There exists a representation $\rho: \operatorname{sl}(2) \oplus \operatorname{sl}(2) \rightarrow \operatorname{End}\left(I H^{*}\left(\mathfrak{N}_{\beta}\right)\right)$ in such a way that the restricted representations $\rho_{L}, \rho_{R}, \rho_{D}: \operatorname{sl}(2) \rightarrow \operatorname{End}\left(I H^{*}\left(\mathfrak{N}_{\beta}\right)\right)$ associated respectively with the first, the second and the diagonal sl(2) subalgebras of $\operatorname{sl}(2) \oplus \operatorname{sl}(2)$ have the following interpretations: $\rho_{L}$ resp. $\rho_{R}$ corresponds to the Lefschetz action in the "fiber resp. base direction" of the cohomologies of $\mathfrak{N}_{\beta} \rightarrow S_{\beta}$ while $\rho_{D}$ corresponds to the usual Lefschetz action. Hence one can introduce

$$
\Lambda_{\beta}(y):=\operatorname{Tr}_{I H^{*}\left(\mathfrak{N}_{\beta}\right)}(-1)^{H_{D}} y^{H_{L}} \in \mathbb{Z}\left[y, y^{-1}\right],
$$

where $H_{L}$ and $H_{D}$ are respectively the images by $\rho_{L}$ and $\rho_{D}$ of the Cartan generator of $\operatorname{sl}(2)$. (The eigenvalues of $H_{L}$ and $H_{D}$ are twice spins.) Note the obvious symmetry $\Lambda_{\beta}\left(y^{-1}\right)=\Lambda_{\beta}(y)$.

According to [13], this claim is true if $\mathfrak{N}_{\beta} \rightarrow S_{\beta}$ is a projective morphism between two normal projective varieties.

In the rest of the paper we only consider the cases when $\beta$ 's are of simple class. Hence $\mathfrak{M}_{\beta, d}$ and $\mathfrak{N}_{\beta}$ do exist and they are smooth and connected. When dealing with $\mathfrak{N}_{\beta}$ we do not need $I H^{*}(-)$ and $H^{*}(-)$ will suffice.

Gopakumar and Vafa argued that $\Lambda_{\beta}(y)$ 's determine the Gromov-Witten potentials of $X$. In particular, considering certain combinations of irreducible representations of $\operatorname{sl}(2)$ to be fundamental, they expanded $\Lambda_{\beta}(y)$ as

$$
\epsilon(\beta) \Lambda_{\beta}(y)=\sum_{h=0}^{g(\beta)} N_{\beta}^{h}\left(y^{\frac{1}{2}}-y^{-\frac{1}{2}}\right)^{2 h} .
$$

Then they considered that the integers $N_{\beta}^{h}$ are alternative fundamental invariants $^{4}$. Since $H_{D}$ acts as the multiplication of $\operatorname{dim} \mathfrak{N}_{\beta}-k$ on $H^{k}\left(\mathfrak{N}_{\beta}\right)$, we have $\epsilon(\beta) \Lambda_{\beta}(1)=\chi\left(\mathfrak{N}_{\beta}\right)=N_{\beta}^{0}$.

This claim of Gopakumar and Vafa may sound somewhat at odds with our earlier statement: Considering D2-branes alone is insufficient. Actually they mapped the problem into $M$-theory and used a physical interpretation of space-time effective theory typically using Schwinger-like computations. This is the reason why $D 0$-branes secretly sneak in interprested in terms of type IIA theory.

The consistency of our proposal with that of Gopakumar and Vafa implies

Conjecture 7.1.

$$
f_{\beta}(y)=\frac{\epsilon(\beta) \Lambda_{\beta}(y)}{\left(y^{\frac{1}{2}}-y^{-\frac{1}{2}}\right)^{2}} .
$$

\footnotetext{
${ }^{4}$ Their definition of the invariants differ in signs with $N_{\beta}^{h}$ here.
} 
Remark. Since $\mathfrak{N}_{0}=S_{0}=X$ and the "fiber direction" is void, we have $\Lambda_{0}(y)=-\chi(X)$. Consequently (7.3) is true for $\beta=0$.

Despite its innocent looking the geometrical implication of (7.3) is rather non-trivial since the interpretations are quite different on both sides of the equation.

\section{§8. Inversion Relations}

Before discussing the validity of (7.3) let us pause to study purely algebraic consequences of (7.3). We expand $\iota_{ \pm} f_{\beta}(y)$ as (5.2) and $\Lambda_{\beta}(y)$ as (7.2). If we set

$$
g=g(\beta), \quad e_{d}=\chi\left(\mathfrak{M}_{\beta, d}\right), \quad m_{i}=N_{\beta}^{g(\beta)-i},
$$

then the following proposition gives the explicit relations between $\chi\left(\mathfrak{M}_{\beta, d}\right)$ and $N_{\beta}^{h}$. Note that since $e_{0}=\chi\left(S_{\beta}\right)$ by our definition, the relation $e_{0}=m_{0}$ below implies $N_{\beta}^{g(\beta)}=\chi\left(S_{\beta}\right)$.

Proposition 8.1. Let $g \geq 0$ be an integer. Suppose that sequences of numbers $\left\{e_{d}\right\}_{d=0}^{\infty}$ and $\left\{m_{i}\right\}_{i=0}^{g}$ are related by

$$
\sum_{d=0}^{\infty} e_{d} y^{ \pm(d+1-g)}=\iota_{ \pm} \sum_{i=0}^{g} m_{i}\left(y^{\frac{1}{2}}-y^{-\frac{1}{2}}\right)^{2(g-i)-2} .
$$

(A) One can express $\left\{e_{d}\right\}_{d=0}^{\infty}$ in terms of $\left\{m_{i}\right\}_{i=0}^{g}$ as:

$* g=0$ :

$$
e_{d}=(d+1) m_{0}, \quad(d \geq 0),
$$

* $g=1$ :

$$
\begin{aligned}
& e_{0}=m_{0}, \\
& e_{d}=d m_{1}, \quad(d \geq 1),
\end{aligned}
$$

* $g \geq 2$ :

$$
\begin{array}{rlr}
e_{d}= & \sum_{i=0}^{d}\left(\begin{array}{cr}
d+i+1-2 g \\
d-i
\end{array}\right) m_{i}, & (g-1 \geq d \geq 0), \\
e_{d}= & \sum_{i=0}^{2 g-2-d}\left(\begin{array}{c}
d+i+1-2 g \\
d-i
\end{array}\right) m_{i} & \\
& +(d+1-g) m_{g}, & (2 g-2 \geq d \geq g), \\
e_{d}= & (d+1-g) m_{g}, & (d \geq 2 g-1) .
\end{array}
$$


(B) Conversely, $\left\{m_{i}\right\}_{i=0}^{g}$ can be expressed in terms of $\left\{e_{d}\right\}_{d=0}^{g}$ as:

* $g=0$ :

* $g=1$ :

$$
m_{0}=e_{0}, \quad m_{1}=e_{1},
$$

* $g \geq 2$ :

$$
\begin{aligned}
& m_{0}=e_{0}, \\
& m_{i}=e_{i}+\sum_{d=0}^{i-1} \frac{2 g-2 d-2}{i-d}\left(\begin{array}{c}
2 g-i-d-3 \\
i-d-1
\end{array}\right) e_{d}, \\
& m_{g}=e_{g}-e_{g-2} . \quad(g-1 \geq i \geq 1),
\end{aligned}
$$

(C) If $\left\{e_{d}\right\}_{d=0}^{\infty}$ are integers, so are $\left\{m_{i}\right\}_{i=0}^{g}$ and vice versa.

Proof. (8.2) implies the following Taylor expansion at $y=0$ :

$$
\sum_{d=0}^{\infty} e_{d} y^{d}=\sum_{i=0}^{g} m_{i} y^{i}(1-y)^{2(g-i)-2} .
$$

It suffices to analyze this equation.

(A) This is a consequence of straightforward binomial expansions of the right hand side of (8.14).

(B) Since the cases $g=0$ and $g=1$ are obvious, we assume $g \geq 2$. Notice that (8.14) is equivalent to

$$
\frac{\sum_{d=0}^{\infty} e_{d} y^{d}}{(1-y)^{2 g-2}}=\sum_{i=0}^{g} m_{i}\left(\frac{y}{(1-y)^{2}}\right)^{i} .
$$

Then, by the Lagrange inversion formula, one finds

$$
\begin{aligned}
m_{i} & =\operatorname{Res}_{y=0}\left[\frac{\sum_{d=0}^{\infty} e_{d} y^{d}}{(1-y)^{2 g-2}}\left(\frac{y}{(1-y)^{2}}\right)^{\prime}\left(\frac{y}{(1-y)^{2}}\right)^{-i-1}\right] \\
& =\operatorname{Res}_{y=0}\left[\frac{1+y}{(1-y)^{2(g-i)-1}} \sum_{d=0}^{\infty} e_{d} y^{d-i-1}\right],
\end{aligned}
$$

where' represents the derivative with respect to $y$. To evaluate this residue it is better to proceed case by case: 
* Suppose that $2(g-i)-1 \leq 0$. Then we actually have to have $g=i$ and

$$
m_{g}=\operatorname{Res}_{y=0}\left[\left(1-y^{2}\right) \sum_{d=0}^{\infty} e_{d} y^{d-g-1}\right] .
$$

It is straightforward to evaluate this residue. The result is given by (8.13).

* Suppose instead that $2(g-i)-1>0$. One uses the formula

$$
\frac{1}{(1-y)^{k}}=\sum_{n=0}^{\infty}\left(\begin{array}{c}
k+n-1 \\
n
\end{array}\right) y^{n}
$$

(valid for a positive integer $k$ ) to obtain

$$
m_{i}=\operatorname{Res}_{y=0}\left[(1+y) \sum_{d, n=0}^{\infty}\left(\begin{array}{c}
2(g-i)+n-2 \\
n
\end{array}\right) e_{d} y^{n+d-i-1}\right] .
$$

If $i=0$ one easily sees that $m_{0}=e_{0}$. If $i \geq 1$, the evaluation of the residue gives

$$
m_{i}=\sum_{d=0}^{i}\left(\begin{array}{c}
2 g-i-d-2 \\
i-d
\end{array}\right) e_{d}+\sum_{d=0}^{i-1}\left(\begin{array}{c}
2 g-i-d-3 \\
i-d-1
\end{array}\right) e_{d}
$$

which can be rewritten as (8.12).

(C) This is already obvious from what we have seen in this proof.

\section{$\S 9 . \quad D 2-D 0$ as Vortices}

We briefly recall the vortex picture of $D 2-D 0$ branes. For more on this see [21]. Let $C \subset X$ be a smooth irreducible curve of genus $g$. Suppose that a $D 2$-brane is singly wrapping around $C$ and $d D 0$-branes are bound to it. Such a BPS system may be identified with vortices on $C$ with magnetic flux $d$. As well-known the moduli space of such vortices is the $d$-th symmetric product of $C$ which we write as $C^{(d)}$. There exists a classical result by Macdonald on the Euler-Poincaré characteristics of symmetric products of smooth curves. As argued in [21], by taking into account the fact that $D$-brane charges must be measured by Mukai vectors, it is more natural to consider a twisted version of Macdonald formula:

$$
\sum_{d=0}^{\infty} \chi\left(C^{(d)}\right) y^{ \pm(d+1-g)}=\iota_{ \pm}\left(y^{\frac{1}{2}}-y^{-\frac{1}{2}}\right)^{2 g-2} .
$$


A naive way to treat $D 2-D 0$ branes in more general settings is to extend the vortex picture relatively and to replace $C^{(d)}$ by $\mathcal{C}_{\beta}^{[d]}$. To simplify the situation considerably we make a rather strong

Assumption 9.1. All the fibers of $\mathcal{C}_{\beta} \rightarrow S_{\beta}$ are integral. We have $\mathfrak{M}_{\beta, d}=$ $\mathcal{C}_{\beta}^{[d]}$ and $\mathfrak{N}_{\beta}=\overline{\mathcal{J}}_{\beta}$.

Hence,

$$
\sum_{d=0}^{\infty} \chi\left(\mathcal{C}_{\beta}^{[d]}\right) y^{ \pm(d+1-g(\beta))}=\iota_{ \pm} f_{\beta}(y) .
$$

From this (5.16) immediately follows.

Consider for instance the case of $\mathcal{O}_{\mathbb{P}^{1}}(-1) \oplus \mathcal{O}_{\mathbb{P}^{1}}(-1)$. Let $\left[\mathbb{P}^{1}\right]$ denote the class of the $\mathbb{P}^{1}$. Then,

$$
f_{\left[\mathbb{P}^{1}\right]}(y)=\left(y^{\frac{1}{2}}-y^{-\frac{1}{2}}\right)^{-2},
$$

and $f_{n\left[\mathbb{P}^{1}\right]}(y)=0$ for $n>1$. This expression can also be obtained (in a difficult way!) either by Gromov-Witten theory [4] or by the topological vertex formalism [1]. In $\S 2$ we said that bubble trees in Gromov-Witten theory turn into $D$ 0-branes (vortices). It is instructive to see how this happens on this simple example. In [4], the authors use the localization technique to evaluate the Gromov-Witten invariants. This boils down to calculate the bubbling contributions at the north and south poles of the $\mathbb{P}^{1}$. On the other hand, the approach of the topological vertex gives a diagram consisting of one internal edge corresponding to the $\mathbb{P}^{1}$ and two trivalent vertices corresponding to the north and south poles. The torus-fixed $D 2-D 0$ configurations relevant to calculate $f_{\left[\mathbb{P}^{1}\right]}(y)$ is such that a $D 2$-brane wraps singly around the $\mathbb{P}^{1}$ (so the $2 d$ Young diagram is a single box) and D0-branes are localized to the north or south poles, i.e. the two vertices.

We also note that (9.3) has an interpretation as a two-point function of vertex operators [12, 28] (See also [21]). This matches well with the appearance of the Schur polynomials in the approach of [1].

In the simplified setting we assume later, we will find that $f_{\beta}(y)$ has an expansion of the form

$$
f_{\beta}(y)=\sum_{h=0}^{g(\beta)} n_{\beta}^{h} \varphi_{h}(y),
$$

with $n_{\beta}^{h} \in \mathbb{Z}$ and $\varphi_{h}(y)$ being a rational function. In fact there arise at least two natural choices of basis functions $\varphi_{h}(y)$. One choice is associated with 
Gopakumar-Vafa's expansion (7.2) and $\varphi_{h}(y)$ takes a simple form. The other choice has a clear geometrical meaning but $\varphi_{h}(y)$ takes a more complicated form.

In [21] we studied an analogous case with $X$ replaced by a projective $K 3$ surface $M$. Corresponding to $\mathcal{C}_{\beta} \rightarrow S_{\beta}$ in the present situation we considered the universal family of curves $\mathcal{C}_{n} \rightarrow\left|D_{n}\right| \cong \mathbb{P}^{n}$ where $D_{n}$ is a smooth (integral) curve of genus $n$ on $M$. Associated to $\mathcal{C}_{n} \rightarrow\left|D_{n}\right|$ one considers the relative compactified Jacobian $\overline{\mathcal{J}}_{n} \rightarrow\left|D_{n}\right|$ together with the Abel-Jacobi map $\mathcal{C}_{n}^{[d]} \rightarrow$ $\overline{\mathcal{J}}_{n}$. With the technical assumptions there, all the fibers of $\mathcal{C}_{n} \rightarrow\left|D_{n}\right|$ are integral and both $\mathcal{C}_{n}^{[d]}$ and $\overline{\mathcal{J}}_{n}$ are smooth. In [21] we proved an exact formula for

$$
\sum_{d=0}^{\infty} \chi\left(\mathcal{C}_{n}^{[d]}\right) y^{ \pm(d+1-n)} .
$$

(The proof there rested on Brill-Noether theory of sheaves on $K 3$ surfaces developed by Yoshioka [36] and Markman [23].)

Remark. In [19], the authors tried to develop an algorithm for calculating the numbers $N_{\beta}^{h}$ and in the process of this they also considered $\mathcal{C}_{\beta}^{[d]}$. However in their treatment it played only an auxiliary role since their point of view is that of Gopakumar-Vafa and not of the $D 2-D 0$ picture. They applied their formalism to concrete examples and obtained satisfactory results with the caveat that one must be careful when there are reducible fibers in the family of curves (e.g. the case of local $\mathbb{P}^{2}$ ). In the following we will see that some of their findings can be uniformly understood once one considers the relation between the Gopakumar-Vafa picture and the $D 2-D 0$ picture.

\section{§10. Abel-Jacobi Maps and Lefschetz Actions}

Let $C$ be a smooth irreducible curve of genus $g$ and let $J(C)$ be its Jacobian. We have

$$
(-1)^{g} \operatorname{Tr}_{H^{*}(J(C))}(-1)^{H} y^{H}=\left(y^{\frac{1}{2}}-y^{-\frac{1}{2}}\right)^{2 g},
$$

where $H$ is the image of the Cartan generator of the Lefschetz $\operatorname{sl}(2)$ as before. Rather trivially, it follows that

$$
\sum_{d=0}^{\infty} \chi\left(C^{(d)}\right) y^{ \pm(d+1-g)}=\iota_{ \pm} \frac{(-1)^{g} \operatorname{Tr}_{H^{*}(J(C))}(-1)^{H} y^{H}}{\left(y^{\frac{1}{2}}-y^{-\frac{1}{2}}\right)^{2}} .
$$

What (7.3) implies is that one may proceed relatively: 
Conjecture 10.1.

$$
\sum_{d=0}^{\infty} \chi\left(\mathcal{C}_{\beta}^{[d]}\right) y^{ \pm(d+1-g(\beta))}=\iota_{ \pm} \frac{\epsilon(\beta) \Lambda_{\beta}(y)}{\left(y^{\frac{1}{2}}-y^{-\frac{1}{2}}\right)^{2}}
$$

This is not obvious at all since there can be singular fibers in $\mathcal{C}_{\beta} \rightarrow S_{\beta}$. However, with some simplifying assumptions, this conjecture can be shown to be true. See Theorem 12.1. Notice that one may take $n_{\beta}^{h}=N_{\beta}^{h}$ and $\varphi_{h}(y)=$ $\left(y^{\frac{1}{2}}-y^{-\frac{1}{2}}\right)^{2 h-2}$ in $(9.4)$.

Conjecture 10.1 implies relations between $\chi\left(\mathcal{C}_{\beta}^{[d]}\right)$ and $N_{\beta}^{h}$ again by Proposition 8.1. In particular, we note that (8.12) coincides with the relation claimed in [19]. If $d \geq 2 g-1$ the Abel-Jacobi map $\mathcal{C}_{\beta}^{[d]} \rightarrow \overline{\mathcal{J}}_{\beta}$ is a fibration with fiber $\mathbb{P}^{d-g}$. Since $m_{g}=N_{\beta}^{0}=\chi\left(\overline{\mathcal{J}}_{\beta}\right)$, the relation $e_{d}=(d+1-g) m_{g}$ is just a reflection of this projective bundle structure.

It is again instructive to see the situation for the $K 3$ surface $M$ as before. There is little harm ${ }^{5}$ in replacing $\overline{\mathcal{J}}_{n}$ by the Hilbert scheme of points $M^{[n]}:=$ Hilb $_{M}^{n}$. When one views $M^{[n]}$ as a compact hyperkähler manifold ${ }^{6}$ of real dimension $4 n$, there are a triple of Kähler forms $\omega_{I}, \omega_{J}, \omega_{K}$ and therefore a triple of Lefschetz actions $\rho_{I}, \rho_{J}, \rho_{K}: \operatorname{sl}(2) \rightarrow \operatorname{End}\left(H^{*}\left(M^{[n]}\right)\right)$. According to Verbitsky [33], $\rho_{I}, \rho_{J}, \rho_{K}$ generate an action of $s o(4,1)$ on $H^{*}\left(M^{[n]}\right)$. Gopakumar-Vafa's $s l(2) \oplus \operatorname{sl}(2)$ should be identified (with the replacement of $\overline{\mathcal{J}}_{n}$ by $M^{[n]}$ understood) as a subalgebra of Verbitsky's $s o(4,1)$.

We choose one complex structure on $M^{[n]}$ thereby viewing it as an irreducible holomorphic symplectic manifold of complex dimension $2 n$. Denote the associated Kähler form by $\varpi$. Then $\rho_{D}: \operatorname{sl}(2) \rightarrow \operatorname{End}\left(H^{*}\left(M^{[n]}\right)\right)$ should be identified with the usual Lefschetz action. So the lowering operator of the $s l(2)$ is represented by the wedge operation of $\varpi$ and the raising one by its adjoint. On the other hand, as we will justify shortly, $\rho_{L}: \operatorname{sl}(2) \rightarrow \operatorname{End}\left(H^{*}\left(M^{[n]}\right)\right)$ should be identified with the holomorphic Lefschetz action first considered by Fujiki [8]. So the lowering operator is represented by the wedge operation of a holomorphic 2-form $\sigma \in H^{0}\left(M^{[n]}, \Omega_{M^{[n]}}^{2}\right)$ and the raising one by its adjoint.

Apparently an analog of $\Lambda_{\beta}(y)$ is

$$
\Lambda_{n}(y):=\operatorname{Tr}_{H^{*}\left(M^{[n]}\right)}(-1)^{H_{D}} y^{H_{L}} .
$$

Proposition 10.1 (Thompson's observation). With the convention that $\chi_{y}(-)$ is meant for $\chi_{-y}(-)$ in the original sense of Hirzebruch, we have

$$
\Lambda_{n}(y)=y^{-n} \chi_{y}\left(M^{[n]}\right) .
$$

\footnotetext{
${ }^{5}$ As well-known they are birationally equivalent or even deformation equivalent under the conditions in [21]. See there and references therein.

${ }^{6}$ See $[16,17]$ for surveys.
} 
Proof. Since $H_{D}$ acts as the multiplication of $2 n-p-q$ on $H^{q}\left(M^{[n]}, \Omega_{M^{[n]}}^{p}\right)$ and we have $(-1)^{2 n-p-q}=(-1)^{p+q}$, we see that $\Lambda_{n}(y)$ is precisely what Thompson writes as $\operatorname{STr} U$ in [32] with $y$ being one of the eigenvalue of $U \in$ $S L(2, \mathbb{C})$. (Amusingly, $\mathrm{S} \operatorname{Tr} U$ is the Rozansky-Witten invariant of a mapping torus $T_{U}^{3}$ if $U \in S L(2, \mathbb{Z})$.) So $(10.5)$ is just a consequence of his observation.

To our please we showed in [21] that

\section{Theorem 10.1.}

$$
\sum_{d=0}^{\infty} \chi\left(\mathcal{C}_{n}^{[d]}\right) y^{ \pm(d+1-n)}=\iota_{ \pm} \frac{y^{-n} \chi_{y}\left(M^{[n]}\right)}{\left(y^{\frac{1}{2}}-y^{-\frac{1}{2}}\right)^{2}}
$$

(This was proved by comparing our formula for $\chi\left(\mathcal{C}_{n}^{[d]}\right)$ and the formula for $\chi_{y}\left(M^{[n]}\right)$ proved earlier by Göttsche and Soergel [9].) Note that what corresponds to $\epsilon(\beta)$ is $(-1)^{2 n}=1$.

\section{$\S 11$. Hilbert Schemes of Points on Nodal Curves}

As a preparation for the next section we make a digression to study Hilbert schemes of points on nodal curves. It is well-known that $C^{[d]}:=\operatorname{Hilb}_{C}^{d}$ coincides with $C^{(d)}$ if $C$ is smooth. This, however, is not the case when $C$ is singular. Suppose that $C_{g, \delta}$ is an integral curve of arithmetic genus $g$ having $\delta$ nodes and no other singularities. In this case we say that $C_{g, \delta}$ is $\delta$-nodal. Note that $g \geq \delta \geq 0$. Let $\nu: \tilde{C}_{g, \delta} \rightarrow C_{g, \delta}$ be the normalization. Then $\chi\left(C_{g, \delta}\right)=$ $\chi\left(\tilde{C}_{g, \delta}\right)-\delta=2-2(g-\delta)-\delta=2+\delta-2 g$. In general

$$
\chi\left(C_{g, \delta}^{(d)}\right)=\left(\begin{array}{c}
d+1+\delta-2 g \\
d
\end{array}\right) .
$$

For the Hilbert schemes we have to find the necessary correction terms to this formula, which we now turn to.

We introduce the standard notation for multinomial coefficients.

$$
\left(\begin{array}{c}
a_{1}+\cdots+a_{n} \\
a_{1}, \ldots, a_{n}
\end{array}\right):=\frac{\left(a_{1}+\cdots+a_{n}\right) !}{a_{1} ! \cdots a_{n} !}, \quad a_{1} \geq 0, \cdots, a_{n} \geq 0 .
$$

Recall that a partition is any sequence $\lambda=\left(\lambda_{1}, \lambda_{2}, \ldots\right)$ of non-negative integers in non-increasing order and containing only finitely many non-zero terms. Then $|\lambda|=\sum_{i} \lambda_{i}$ is the weight of $\lambda$ and we say $\lambda$ is a partition of $d$ if $|\lambda|=d$. When $\lambda$ is a partition of $d$, we also use an alternative notation $\lambda=\left(1^{\delta_{1}} 2^{\delta_{2}} \cdots d^{\delta_{d}}\right)$ where $\delta_{\ell}=\#\left\{i \mid \lambda_{i}=\ell\right\}$ is the multiplicity of $\ell$ in $\lambda$. (Note that $\delta_{\ell}$ might be zero.) We set $\check{\delta}_{1}=\delta_{2}+\cdots+\delta_{d}$ for convenience. 
If $\lambda=\left(1^{\delta_{1}} 2^{\delta_{2}} \cdots d^{\delta_{d}}\right)$ is a partition of $d$, we define $A_{\lambda}$ by

$$
A_{\lambda}=1, \quad(d=0,1), \quad A_{\lambda}=\prod_{\ell=2}^{d}(\ell-1)^{\delta_{\ell}}, \quad(d>1) .
$$

\section{Proposition 11.1.}

$$
\chi\left(C_{g, \delta}^{[d]}\right)=\sum_{\substack{\lambda=\left(1^{\delta_{1} \ldots} d^{\delta} d\right) \\
|\lambda|=d, \check{\delta}_{1} \leq \delta}} A_{\lambda}\left(\begin{array}{c}
\delta \\
\delta-\check{\delta}_{1}, \delta_{2}, \ldots, \delta_{d}
\end{array}\right)\left(\begin{array}{c}
\delta_{1}+1+\delta-\check{\delta}_{1}-2 g \\
\delta_{1}
\end{array}\right) .
$$

Proof. We assume $d>1$ since otherwise the assertion is trivial.

Let $\mathcal{N}$ be the zero-dimensional subscheme of the nodes on $C_{g, \delta}$ so that its length is $\# \mathcal{N}=\delta$. For a given partition $\lambda=\left(1^{\delta_{1}} \cdots d^{\delta_{d}}\right)$ such that $\delta \geq \check{\delta}_{1}$ we partition $\mathcal{N}$ into mutually disjoint union $\mathcal{N}=\coprod_{\ell=1}^{d} \mathcal{N}_{\ell}^{\lambda}$ with $\# \mathcal{N}_{1}^{\lambda}=\delta-\check{\delta}_{1}$ and $\# \mathcal{N}_{\ell}^{\lambda}=\delta_{\ell}(\ell>1)$. In $C_{g, \delta}^{(d)}$ we consider configurations where a multiplicity $\ell$ point collides with each node of $\mathcal{N}_{\ell}^{\lambda}$ for each $\ell>1$. Then $C_{g, \delta}^{[d]}$ differs from $C_{g, \delta}^{(d)}$ in that at each node of $\mathcal{N}_{\ell}^{\lambda}$ a multiplicity $\ell$ point is replaced by the punctual Hilbert scheme of length $\ell$.

After some mental exercise, $\chi\left(C_{g, \delta}^{[d]}\right)$ is then found to be

$$
\sum_{\substack{\lambda=\left(1^{\delta_{1} \ldots d^{\delta} d}\right) \\|\lambda|=d, \check{\delta}_{1} \leq \delta}} \sum_{\mathcal{N}=\coprod_{\ell=1}^{d} \mathcal{N}_{\ell}^{\lambda}} \chi\left(\left(C_{g, \delta} \backslash \coprod_{\ell=2}^{d} \mathcal{N}_{\ell}^{\lambda}\right)^{\left(\delta_{1}\right)}\right) \prod_{\ell=2}^{d}\left(\chi\left(H_{\ell}\right)-1\right)^{\delta_{\ell}},
$$

where $H_{\ell}$ is the punctual Hilbert scheme of length $\ell$ supported at a node. To proceed we have to calculate $\chi\left(H_{\ell}\right)$. According to Ran [30], $H_{\ell}$ is a rational chain

$$
H_{\ell} \cong R_{1} \underset{p_{1}}{\cup} R_{2} \underset{p_{2}}{\cup \cdots} \mathcal{p}_{\ell-2} R_{\ell-1}
$$

where $R_{1}, \ldots, R_{\ell-1}$ are $\mathbb{P}^{1}$ 's and $p_{1}, \ldots, p_{\ell-2}$ are nodes. Therefore $\chi\left(H_{\ell}\right)=$ $(\ell-1) \times 2-(\ell-2)=\ell$ and we understand why the factor $A_{\lambda}$ arises.

Next we easily see that

$$
\chi\left(\left(C_{g, \delta} \backslash \coprod_{\ell=2}^{d} \mathcal{N}_{\ell}^{\lambda}\right)^{\left(\delta_{1}\right)}\right)=\left(\begin{array}{c}
\delta_{1}+1+\delta-\check{\delta}_{1}-2 g \\
\delta_{1}
\end{array}\right) .
$$

Finally, it should be noticed that there are $\left(\underset{\delta-\check{\delta}_{1}, \delta_{2}, \ldots, \delta_{d}}{\delta}\right)$ ways to partition $\mathcal{N}$ into $\coprod_{\ell=1}^{d} \mathcal{N}_{\ell}^{\lambda}$. 
The above formula of $\chi\left(C_{g, \delta}^{[d]}\right)$ is rather complicated and not that convenient in practice. Actually there exists a neat formula for the generating function as we explain below. However, before going to that, it will be worthwhile (and fun!) to look at several examples of this formula:

$$
\begin{aligned}
& \chi\left(C_{g, \delta}\right)=2+\delta-2 g \\
& \chi\left(C_{g, \delta}^{[2]}\right)=\left(\begin{array}{c}
3+\delta-2 g \\
2 \\
日
\end{array}\right)+\quad \delta \\
& \chi\left(C_{g, \delta}^{[3]}\right)=\left(\begin{array}{c}
4+\delta-2 g \\
3 \\
\text { 日 }
\end{array}\right)+\delta(1+\delta-2 g)+2 \delta
\end{aligned}
$$

These agree with the sample computations of [19]. We can give further examples:

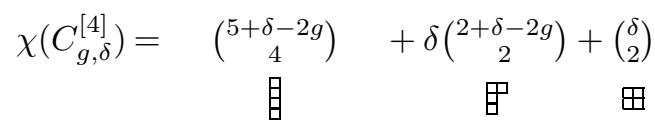

$$
\begin{aligned}
& +2 \delta(1+\delta-2 g)+\quad 3 \delta \\
& \text { एण } \\
& \chi\left(C_{g, \delta}^{[5]}\right)=\left(\begin{array}{c}
6+\delta-2 g \\
5
\end{array}\right)+\delta\left(\begin{array}{c}
3+\delta-2 g \\
3
\end{array}\right)+\left(\begin{array}{l}
\delta \\
2
\end{array}\right)(\delta-2 g) \\
& \text { 臬田田 } \\
& +2 \delta\left(\begin{array}{c}
2+\delta-2 g \\
2
\end{array}\right)+2 \delta(\delta-1)+3 \delta(1+\delta-2 g) \\
& \text { 田罚 } \\
& +\quad 4 \delta \\
& \chi\left(C_{g, \delta}^{[6]}\right)=\left(\begin{array}{c}
7+\delta-2 g \\
6
\end{array}\right)+\delta\left(\begin{array}{c}
4+\delta-2 g \\
4
\end{array}\right)+\left(\begin{array}{l}
\delta \\
2
\end{array}\right)\left(\begin{array}{c}
1+\delta-2 g \\
2
\end{array}\right) \\
& \text { 自目白电 } \\
& +\quad\left(\begin{array}{l}
\delta \\
3
\end{array}\right) \quad+2 \delta\left(\begin{array}{c}
3+\delta-2 g \\
3
\end{array}\right)+2 \delta(\delta-1)(\delta-2 g) \\
& \text { 田四田 } \\
& +\quad 2^{2}\left(\begin{array}{l}
\delta \\
2
\end{array}\right) \quad+3 \delta\left(\begin{array}{c}
2+\delta-2 g \\
2
\end{array}\right)+\quad 3 \delta(\delta-1) \\
& \text { 巴 四 四 } \\
& +4 \delta(1+\delta-2 g)+\quad 5 \delta \\
& \text { मسים }
\end{aligned}
$$


Let us now turn to the generating function:

\section{Proposition 11.2.}

$$
\sum_{d=0}^{\infty} \chi\left(C_{g, \delta}^{[d]}\right) y^{ \pm(d+1-g)}=\iota_{ \pm}\left(y^{\frac{1}{2}}-y^{-\frac{1}{2}}\right)^{2 g-2}\left(1+\left(y^{\frac{1}{2}}-y^{-\frac{1}{2}}\right)^{-2}\right)^{\delta} .
$$

Proof. By eliminating the sum over $\delta_{1}$ in (11.4) one obtains that

$$
\begin{aligned}
\chi\left(C_{g, \delta}^{[d]}\right)= & \sum_{\substack{\delta_{2} \geq 0, \ldots, \delta_{d} \geq 0 \\
\sum_{\ell \geq 2} \ell \delta_{\ell} \leq d \\
\sum_{\ell \geq 2} \delta_{\ell} \leq \delta}}\left(\begin{array}{c}
\delta \\
\sum_{\ell \geq 2} \delta_{\ell}
\end{array}\right)\left(\begin{array}{c}
\sum_{\ell \geq 2} \delta_{\ell} \\
\delta_{2}, \ldots, \delta_{d}
\end{array}\right)\left(\prod_{\ell=2}^{d}(\ell-1)^{\delta_{\ell}}\right) \\
& \times\left(\begin{array}{c}
d-\sum_{\ell \geq 2} \ell \delta_{\ell}+1+\delta-\sum_{\ell \geq 2} \delta_{\ell}-2 g \\
d-\sum_{\ell \geq 2} \ell \delta_{\ell}
\end{array}\right) .
\end{aligned}
$$

Then consideration of the generating function leads to

$$
\begin{aligned}
\sum_{d=0}^{\infty} \chi\left(C_{g, \delta}^{[d]}\right) y^{d}=\sum_{k=0}^{\delta} \sum_{\substack{\delta_{2} \geq 0, \delta_{3} \geq 0, \ldots \\
k=\sum_{\ell \geq 2} \delta_{\ell}}}\left(\begin{array}{l}
\delta \\
k
\end{array}\right)\left(\begin{array}{c}
k \\
\delta_{2}, \delta_{3}, \ldots
\end{array}\right) \\
\\
\times\left(\prod_{\ell \geq 2}(\ell-1)^{\delta_{\ell}}\right) y^{\sum_{\ell \geq 2} \ell \delta_{\ell}}(1-y)^{2 g-\delta+k-2}
\end{aligned}
$$

where we understand the sequence $\delta_{2}, \delta_{3}, \ldots$ contains only finitely many nonzero terms. The right hand side can be rewritten as

$$
\begin{aligned}
& (1-y)^{2 g-\delta-2} \sum_{k=0}^{\delta}\left(\begin{array}{l}
\delta \\
k
\end{array}\right) \\
& \times \sum_{\substack{\delta_{2} \geq 0, \delta_{3} \geq 0, \ldots \\
k=\sum_{\ell \geq 2} \delta_{\ell}}}\left(\begin{array}{c}
k \\
\delta_{2}, \delta_{3}, \ldots
\end{array}\right) \prod_{\ell \geq 2}\left\{(\ell-1) y^{\ell}(1-y)\right\}^{\delta_{\ell}} .
\end{aligned}
$$

The multinomial theorem simplifies this as

$$
(1-y)^{2 g-\delta-2} \sum_{k=0}^{\delta}\left(\begin{array}{l}
\delta \\
k
\end{array}\right)\left(\sum_{\ell \geq 2}(\ell-1) y^{\ell}(1-y)\right)^{k} .
$$

By summing up the geometric series one obtains

$$
(1-y)^{2 g-\delta-2} \sum_{k=0}^{\delta}\left(\begin{array}{l}
\delta \\
k
\end{array}\right)\left(\frac{y^{2}}{1-y}\right)^{k}
$$


By the binomial theorem this is equal to

$$
(1-y)^{2 g-\delta-2}\left(1+\frac{y^{2}}{1-y}\right)^{\delta} .
$$

It thus follows that

$$
\sum_{d=0}^{\infty} \chi\left(C_{g, \delta}^{[d]}\right) y^{d}=(1-y)^{2 g-2}\left(1+\frac{y}{(1-y)^{2}}\right)^{\delta} .
$$

To see that this leads to (11.8) is easy.

Let $J\left(C_{g, \delta}\right)$ be the generalized Jacobian of $C_{g, \delta}$ and $\overline{J\left(C_{g, \delta}\right)}$ its compactification.

\section{Proposition 11.3.}

$$
(-1)^{g} \operatorname{Tr}_{H^{*}\left(\overline{J\left(C_{g, \delta}\right)}\right)}(-1)^{H} y^{H}=\left(y^{\frac{1}{2}}-y^{-\frac{1}{2}}\right)^{2(g-\delta)}\left(\left(y^{\frac{1}{2}}-y^{-\frac{1}{2}}\right)^{2}+1\right)^{\delta} .
$$

Proof. This is (with a correction of sign) implicit in [19]. (See the discussion around eq. (5.8) of [19].) We have an exact sequence of commutative algebraic groups ${ }^{7}$

$$
1 \rightarrow\left(\mathbb{C}^{\times}\right)^{\delta} \rightarrow J\left(C_{g, \delta}\right) \stackrel{\nu^{*}}{\longrightarrow} J\left(\tilde{C}_{g, \delta}\right) \rightarrow 1 .
$$

Hence $\overline{J\left(C_{g, \delta}\right)}$ is the product of $J\left(\tilde{C}_{g, \delta}\right)$ and the compactification of $\left(\mathbb{C}^{\times}\right)^{\delta}$. The latter is given by

$$
\left(\mathbb{P}^{1}\right)^{\delta} / \sim \cong\left(C_{*}\right)^{\delta},
$$

where $\sim$ is a certain equivalence relation essentially identifying 0 and $\infty$ of each $\mathbb{P}^{1}$ and $C_{*}$ is a rational curve with a single node. See IIIb, $\S 5$ in [27] for an exposition. Since $C_{*}$ is obtained by pinching a homologically non-trivial 1-cycle from an elliptic curve, we obtain

$$
(-1) \operatorname{Tr}_{H^{*}\left(C_{*}\right)}(-1)^{H} y^{H}=\left(y^{\frac{1}{2}}-y^{-\frac{1}{2}}\right)^{2}+1 .
$$

Combining this with

$$
(-1)^{g-\delta} \operatorname{Tr}_{H^{*}\left(J\left(\tilde{C}_{g, \delta}\right)\right)}(-1)^{H} y^{H}=\left(y^{\frac{1}{2}}-y^{-\frac{1}{2}}\right)^{2(g-\delta)},
$$

we easily obtain (11.16).

The following may be viewed as an extension of (10.2).

\footnotetext{
${ }^{7}$ A line bundle on $C_{g, \delta}$ can be obtained from one on $\tilde{C}_{g, \delta}$ by gluing the fibers over those points on $\tilde{C}_{g, \delta}$ which are identified to form nodes on $C_{g, \delta}$. For each node the ways to glue two lines are parametrized by $G L(1, \mathbb{C})=\mathbb{C}^{\times}$.
} 
Theorem 11.1.

$$
\sum_{d=0}^{\infty} \chi\left(C_{g, \delta}^{[d]}\right) y^{ \pm(d+1-g)}=\iota_{ \pm} \frac{(-1)^{g} \operatorname{Tr}_{H^{*}}\left(\overline{J\left(C_{g, \delta}\right)}(-1)^{H} y^{H}\right.}{\left(y^{\frac{1}{2}}-y^{-\frac{1}{2}}\right)^{2}} .
$$

Proof. An immediate consequence of Proposition 11.2 and Proposition 11.3.

\section{$\S 12 . \quad$ Severi Varieties}

We now investigate how the fibration structure of $\mathcal{C}_{\beta} \rightarrow S_{\beta}$ is directly reflected to properties of $f_{\beta}(y)$. We start by

Definition 12.1. The Severi variety of $\delta$-nodal curves is defined by

$$
V_{\beta, \delta}=\left\{x \in S_{\beta} \mid\left(\mathcal{C}_{\beta}\right)_{x} \text { has } \delta \text { nodes and no other singularities }\right\},
$$

where $\left(\mathcal{C}_{\beta}\right)_{x}$ is the fiber over $x$.

Severi varieties were originally studied by Severi (and corrected by Harris) for curves of fixed degrees in $\mathbb{P}^{2}$. There are numerous works on Severi varieties of other surfaces. See for instance $[3,5]$. Here we are extending the definition for Calabi-Yau threefolds assuming an appropriate family of curves $\mathcal{C}_{\beta} \rightarrow S_{\beta}$. For attempts in defining Severi varieties of threefolds see $[6,7]$ and references therein. One of the principal issues in the studies of Severi varieties is whether or not they are regular. The standard way to investigate this is to resort to a deformation theory. There are many works for surfaces and these show that in most cases Severi varieties of surfaces are regular but a care is needed when they are of general type. See again $[3,5]$. If $V_{\beta, \delta}$ is regular, the $\delta$ nodes can be independently smoothed and $\operatorname{dim} V_{\beta, \delta}=\operatorname{dim} S_{\beta}-\delta$. Below we assume the regularity of $V_{\beta, \delta}$ for simplicity so that $\chi\left(V_{\beta, \delta}\right)$ is meaningful.

In order to appreciate the roles of the Severi varieties we wish to add one more strong

Assumption 12.1. All the singular fibers of $\mathcal{C}_{\beta} \rightarrow S_{\beta}$ are nodal. Hence, $S_{\beta}=\coprod_{\delta=0}^{g(\beta)} V_{\beta, \delta}$.

In this ideal situation,

\section{Proposition 12.1.}

$$
\begin{aligned}
& \sum_{d=0}^{\infty} \chi\left(\mathcal{C}_{\beta}^{[d]}\right) y^{ \pm(d+1-g(\beta))} \\
& =\iota_{ \pm} \sum_{\delta=0}^{g(\beta)} \chi\left(V_{\beta, \delta}\right)\left(y^{\frac{1}{2}}-y^{-\frac{1}{2}}\right)^{2 g(\beta)-2}\left(1+\left(y^{\frac{1}{2}}-y^{-\frac{1}{2}}\right)^{-2}\right)^{\delta} .
\end{aligned}
$$


Proof. By our assumptions, we see (as in [35])

$$
\sum_{d=0}^{\infty} \chi\left(\mathcal{C}_{\beta}^{[d]}\right) y^{ \pm(d+1-g(\beta))}=\sum_{\delta=0}^{g(\beta)} \chi\left(V_{\beta, \delta}\right) \sum_{d=0}^{\infty} \chi\left(C_{g(\beta), \delta}^{[d]}\right) y^{ \pm(d+1-g(\beta))},
$$

where $C_{g(\beta), \delta}$ is any integral $\delta$-nodal curve of arithmetic genus $g(\beta)$. Then (12.2) follows immediately from Proposition 11.2.

Notice that (12.2) gives another expansion of the form (9.4). We have now reached the main assertion:

Theorem 12.1. $\quad$ Under our assumptions Conjecture 10.1 is true.

Proof. Observe that

$$
\begin{aligned}
\epsilon(\beta) \Lambda_{\beta}(y) & =\epsilon(\beta) \sum_{\delta=0}^{g(\beta)}(-1)^{\operatorname{dim} S_{\beta}} \chi\left(V_{\beta, \delta}\right) \operatorname{Tr}_{H^{*}\left(J\left(C_{g(\beta), \delta}\right)\right.}(-1)^{H} y^{H} \\
& =\sum_{\delta=0}^{g(\beta)} \chi\left(V_{\beta, \delta}\right)(-1)^{g(\beta)} \operatorname{Tr}_{H^{*}\left(\overline{J\left(C_{g(\beta), \delta}\right)}\right.}(-1)^{H} y^{H}
\end{aligned}
$$

where $C_{g(\beta), \delta}$ is again any integral $\delta$-nodal curve of arithmetic genus $g(\beta)$. Use (12.3), (12.4) and Theorem 11.1.

The relations between $\chi\left(V_{\beta, \delta}\right)$ and $N_{\beta}^{h}$ are as follows:

\section{Proposition 12.2.}

$$
\begin{aligned}
N_{\beta}^{g(\beta)-i} & =\sum_{\delta=i}^{g(\beta)}\left(\begin{array}{l}
\delta \\
i
\end{array}\right) \chi\left(V_{\beta, \delta}\right), \\
\chi\left(V_{\beta, \delta}\right) & =\sum_{i=\delta}^{g(\beta)}(-1)^{i+\delta}\left(\begin{array}{l}
i \\
\delta
\end{array}\right) N_{\beta}^{g(\beta)-i} .
\end{aligned}
$$

In particular, we have

$$
N_{\beta}^{g(\beta)}=\sum_{\delta=0}^{g(\beta)} \chi\left(V_{\beta, \delta}\right)=\chi\left(S_{\beta}\right), \quad N_{\beta}^{0}=\chi\left(V_{\beta, g(\beta)}\right)=\chi\left(\overline{\mathcal{J}}_{\beta}\right) .
$$

Proof. To prove (12.5) it suffices to binomially expand $(\cdots)^{\delta}$ on the right hand side of (12.2) and then notice

$$
\sum_{\delta=0}^{g(\beta)} \sum_{i=0}^{\delta}=\sum_{i=0}^{g(\beta)} \sum_{\delta=i}^{g(\beta)}
$$


The inversion relation that leads to (12.6) is well-known. The last equality in (12.7) is due to a Yau-Zaslow type argument [35].

The way Gopakumar and Vafa introduced $N_{\beta}^{h}$ was representation theoretic and was driven by the motivation of finding a convenient expansion basis as in (7.2). However, to the best of my understanding, the direct geometrical characterization of $N_{\beta}^{h}$ has not yet been available. The relation (12.5) seems to give an intuitive feeling about the geometrical meaning of $N_{\beta}^{g(\beta)-i}$. In general, one can produce a node on a curve by pinching a handle or gluing two points. Conversely given a $\delta$-nodal curve of arithmetic genus $g(\beta)$ one can make a smooth curve of genus $g(\beta)-i$, where $i \leq \delta$, by ungluing (or partially normalizing) $i$ nodes and unpinching $\delta-i$ nodes. Of course there are $\left(\begin{array}{l}\delta \\ i\end{array}\right)$ ways to choose such $i$ nodes from the entire nodes of the curve.

Remark. If $V_{\beta, \delta}=\emptyset$ for all $\delta>i$ then $N_{\beta}^{g(\beta)-i}=\chi\left(V_{\beta, i}\right)$. This was the claim in [19]. However, such an assumption depends on $i$ and cannot be satisfied for all $i$ simultaneously unless everything is empty. In general the relation between $N_{\beta}^{h}$ and $\chi\left(V_{\beta, \delta}\right)$ has to be as above.

To summarize, in our extremely ideal situation we can declare any of

$$
\left\{\chi\left(\mathcal{C}_{\beta}^{[d]}\right)\right\}_{d=0}^{g(\beta)}, \quad\left\{N_{\beta}^{h}\right\}_{h=0}^{g(\beta)}, \quad\left\{\chi\left(V_{\beta, \delta}\right)\right\}_{\delta=0}^{g(\beta)},
$$

as a fundamental set of invariants. They can be converted from one to another.

\section{References}

[1] Aganagic, M., Klemm, A., Marino, M. and Vafa, C., The Topological Vertex, hepth/0305132.

[2] Borcherds, R. E., Automorphic forms on $\mathrm{O}_{s+2,2}(\mathbf{R})$ and infinite products, Invent. Math., 120 (1995), 161-213.

[3] Chiantini, L. and Sernesi, E., Nodal curves on surfaces of general type, Math. Ann., 307 (1997), 41-56, alg-geom/9602012.

[4] Faber, C. and Pandharipande, R., Hodge integrals and Gromov-Witten theory, Invent. Math., 139 (2000), 173-199, math.AG/9810173.

[5] Flamini, F., Families of nodal curves on projective surfaces, Tesi di Dottorato (PhD thesis) - Roma "La Sapienza" (1999).

[6] Equivalence of families of singular schemes on threefolds and on ruled fourfolds, Preprint, math.AG/0303308.

[7] _ Families of nodal curves on projective threefolds and their regularity via postulation of nodes, Preprint, math.AG/0202022.

[8] Fujiki, A., On the de Rham cohomology group of a compact Kähler symplectic manifold, Algebraic geometry, Sendai, 1985, Adv. Stud. Pure Math., 10 (1987), 105-165.

[9] Göttsche, L. and Soergel, W., Perverse sheaves and the cohomology of Hilbert schemes of smooth algebraic surfaces, Math. Ann., 296 (1993), 235-245. 
[10] Gopakumar, R. and Vafa, C., M-theory and topological strings-I, Preprint, hepth/9809187.

[11] M-theory and topological strings-II, Preprint, hep-th/9812127.

[12] Grojnowski, I., Instantons and affine algebras I: the Hilbert scheme and vertex operators, Math. Res. Lett., 3 (1996), 275-291, alg-geom/9506020.

[13] Hosono, S., Saito, M.-H. and Takahashi, A., Relative Lefschetz action and BPS state counting, Internat. Math. Res. Notices, 15 (2001), 783-816, math.AG/0105148.

[14] Harvey, J. A. and Moore, G., Algebras, BPS states, and strings, Nucl. Phys. B, 463 (1996), 315-368, hep-th/9510182.

[15] , On the algebras of BPS states, Comm. Math. Phys., 197 (1998), 489-519, hep-th/9609017.

[16] Huybrechts, D., Compact hyper-Kähler manifolds: basic results, Invent. Math., 135 (1999), 63-113; Erratum, ibid., 152 (2003), 209-212.

[17] Compact hyperkähler manifolds, Calabi-Yau manifolds and related geometries (Nordfjordeid, 2001), Springer, 2003, pp. 161-225.

[18] Iqbal, A., Nekrasov, A., Okounkov, A. and Vafa, C., Quantum Foam and Topological Strings, hep-th/0312022.

[19] Katz, S., Klemm, A. and Vafa, C., M-theory, topological strings and spinning black holes, Adv. Theor. Math. Phys., 3 (1999), 1445-1537, hep-th/9910181.

[20] Kawai, T., in preparation.

[21] Kawai, T. and Yoshioka, K., String partition functions and infinite products, Adv. Theor. Math. Phys., 4 (2000), 397-485, hep-th/0002169.

[22] Mariño, M. and Moore, G., Counting higher genus curves in a Calabi-Yau manifold, Nucl. Phys. B, 543 (1999), 592-614, hep-th/9808131.

[23] Markman, E., Brill-Noether duality for moduli spaces of sheaves on $K 3$ surfaces, $J$. Algebraic Geom., 10 (2001), 623-694, math.AG/9901072.

[24] Maulik, D., Nekrasov, N., Okounkov, A. and Pandharipande, P., Gromov-Witten theory and Donaldson-Thomas theory, math.AG/0312059.

[25] Mukai, S., Symplectic structure of the moduli space of sheaves on an abelian or $K 3$ surface, Invent. Math., 77 (1984), 101-106.

[26] - On the moduli space of bundles on $K 3$ surfaces I, Vector bundles on algebraic varieties, (1987) Tata. Inst. Fund. Res., 1987, pp. 341-413.

[27] Mumford, D., Tata Lectures on Theta II, Progr. Math., 43 Birkhäuser, 1984.

[28] Nakajima, H., Lectures on Hilbert schemes of points on surfaces, American Mathematical Society, 1999.

[29] Okounkov, A., Reshetikhin, N. and Vafa, C., Quantum Calabi-Yau and Classical Crystals, hep-th/0309208.

[30] Ran, Z., Geometry on nodal curves, Preprint, math.AG/0210209, (updates and corrections available at math.ucr.edu/ ${ }^{\sim}$ ziv/papers/geonodal.pdf.)

[31] Thomas, R. P., A holomorphic Casson invariant for Calabi-Yau 3-folds, and bundles on K3 fibrations, J. Diff. Geom, 54 (2000), 367-438, math.AG/9806111.

[32] Thompson, G., A geometric interpretation of the $\chi_{y}$ genus on hyper-Kähler manifolds, Comm. Math. Phys., 212 (2000), 649-652, math.AG/9905102.

[33] Verbitsky, M., Action of the Lie algebra of $\mathrm{SO}(5)$ on the cohomology of a hyper-Kähler manifold, Funct. Anal. Appl., 24 (1991), 229-230.

[34] Witten, E., Topological sigma models, Comm. Math. Phys., 118 (1988), 411-449.

[35] Yau, S.-T. and Zaslow, E., BPS states, string duality, and nodal curves on K3, Nucl. Phys. B, 471 (1996), 503-512.

[36] Yoshioka, K., Some examples of Mukai's reflections on K3 surfaces, J. Reine Angew. Math., 515 (1999), 97-123. 